\title{
Erratum to: Signature Theory in Holographic Algorithms
}

\author{
Jin-Yi Cai ${ }^{1} \cdot$ Pinyan $\mathbf{L u}^{2}$
}

Received: 27 September 2015 / Accepted: 30 October 2015 / Published online: 11 November 2015

(C) Springer Science+Business Media New York 2015

\section{Erratum to: Algorithmica (2011) 61:779-816 DOI 10.1007/s00453-009-9383-3}

1. Theorem 6.2 of [4] contains an error. Specifically the condition in Theorem 6.2 is stated as both necessary and sufficient for a signature $G \in\{0,1\}^{n}$ to be realizable as a matchgate signature under all basis transformations $\left[\begin{array}{cc}1 & x \\ 1 & -x\end{array}\right]$. The condition is indeed sufficient, and the proof in Theorem 6.2 for this is valid; the error is the claim that it is also necessary.

On lines 16-17 of page 810, it claims that there must exist some $i$ and $i+1$, "both in $X$ or both out of $X$, and one is in $D$ and the other is out of $D$." The reason given is that $D \neq \emptyset,[n], X, X^{c}$. This is incorrect. It is possible that $D \neq \emptyset,[n], X, X^{c}$, and yet all changes in membership from $i$ to $i+1$ in $D$ are also changes in membership in $X$. This does not contradict $D \neq \emptyset,[n], X, X^{c}$ because there can be changes in membership in $X$ that are not changes in membership in $D$. Here is a counterexample: $n=8, X=\{2,4,6,8\}, S=\{1,2,4,5\}, S^{\prime}=\{1,4,5,6\}, D=S \oplus S^{\prime}=\{2,6\}$. Because of this error, we cannot prove the orthogonality of the coefficient vectors in (41) and thus we cannot deduce $G^{S} G^{S \oplus X}=0$ in (41).

The online version of the original article can be found under doi:10.1007/s00453-009-9383-3.

J.-Y. Cai: Supported by NSF CCF-1217549.

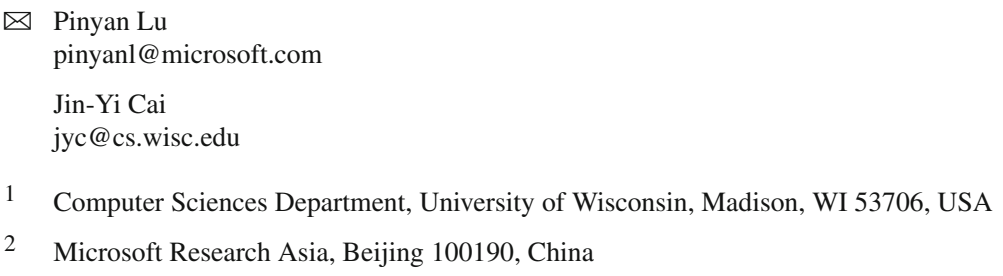


As the condition in Theorem 6.2 is still sufficient, Corollary 6.1 is still valid. All other parts of the paper [4] are correct, and to our knowledge, no other subsequent results of ours and others depend on this part of Theorem 6.2.

2. As pointed out (correctly) on page 806 in [4] that for a signature $G \in\{0,1\}^{n}$, a necessary and sufficient condition for $\left[\begin{array}{cc}1 & x \\ 1 & -x\end{array}\right]^{\otimes n} G$ to be a standard matchgate signature for all $x \neq 0$ is that

- $G^{S}=0$, for all $|S| \neq n / 2$, and

- $H_{2}^{\otimes n} G$ is a standard matchgate signature, where $H_{2}=\left[\begin{array}{cc}1 & 1 \\ 1 & -1\end{array}\right]$.

3. In the following, we give a characterization of the realizability of $\mathrm{G}$ under $\mathrm{H}_{2}$.

Let $\Gamma$ be a matchgate of arity $n$ satisfying $\Gamma^{11 \ldots 1} \neq 0$. We may normalize it to $\Gamma^{11 \ldots 1}=1$. Define an $n \times n$ skew-symmetric matrix $B$ where its $(i, j)$ entry, for $1 \leq i<j \leq n$, is $\Gamma^{11 \ldots 1 \oplus e_{i} \oplus e_{j}}$, the signature value of $\Gamma$ on the bit pattern that has two 1's at the $i$-th and $j$-th bit positions and 0 elsewhere. The theory of matchgate signatures ([3], see also [1,2]) implies that for any $T \subseteq[n], \Gamma^{T}=\operatorname{Pf}(B[T])$, where $\Gamma^{T}$ is the signature value for the bit pattern $T$ denoted by its characteristic sequence, Pf denotes Pfaffian, $B[T]$ is the principal minor of $B$ with all rows and columns in $T$ removed.

Consider the transformation of $\Gamma$ by the Hadamard matrix $\mathrm{H}_{2}$ which is orthogonal up to a scalar $\frac{1}{\sqrt{2}}$. Let $G=H_{2}^{\otimes n} \Gamma$, then for all $S \subseteq[n]$,

$$
G^{S}=\sum_{T \subseteq[n]}(-1)^{|S \cap T|} \Gamma^{T}
$$

If $B$ is any $n \times n$ skew-symmetric matrix, and $\lambda_{1}, \lambda_{2}, \ldots, \lambda_{n}$ are $n$ indeterminants, then Valiant [5] defined the Pfaffian $\operatorname{Sum} \operatorname{PfS}(B)$ to be a polynomial $\sum_{T \subseteq[n]}\left(\prod_{i \in T} \lambda_{i}\right) \operatorname{Pf}(B[T])$.

Now for any $S \subseteq[n]$ we define a sequence of values

$$
\lambda_{i}=\left\{\begin{array}{cl}
-1 & \text { if } i \in S \\
1 & \text { otherwise }
\end{array}\right.
$$

then for these values of $\lambda_{i}$, and the $B$ defined from $\Gamma$,

$$
\operatorname{PfS}(B)=\sum_{T \subseteq[n]}(-1)^{|S \cap T|} \operatorname{Pf}(B[T])=\sum_{T \subseteq[n]}(-1)^{|S \cap T|} \Gamma^{T}=G^{S} .
$$

By the Pfaffian Sum Theorem of [5] this can be expressed as a single Pfaffian. Suppose $n$ is even, then define an $n \times n$ skew-symmetric matrix $\Lambda^{(n)}(S)$ with its $(i, j)$ entry $(-1)^{j-i-1} \lambda_{i} \lambda_{j}=(-1)^{j-i-1+\chi_{S}(i)+\chi_{S}(j)}$ for $1 \leq i<j \leq n$. This matrix has the form

$$
\left[\begin{array}{cccc}
(-1)^{\chi_{S}(1)} & 0 & \ldots & 0 \\
0 & (-1)^{\chi_{S}(2)} & \ldots & 0 \\
\vdots & \vdots & \ddots & \vdots \\
0 & 0 & \ldots & (-1)^{\chi_{S}(n)}
\end{array}\right]\left[\begin{array}{ccccc}
0 & 1 & -1 & \ldots & 1 \\
-1 & 0 & 1 & \ldots & -1 \\
\vdots & \vdots & \vdots & \ddots & \vdots \\
-1 & 1 & -1 & \ldots & 0
\end{array}\right]\left[\begin{array}{cccc}
(-1)^{\chi_{S}(1)} & 0 & \ldots & 0 \\
0 & (-1)^{\chi_{S}(2)} & \ldots & 0 \\
\vdots & \vdots & \ddots & \vdots \\
0 & 0 & \ldots & (-1)^{\chi_{S}(n)}
\end{array}\right]
$$


This is the skew-symmetric matrix with the strict alternating pattern of \pm 1 starting each row with +1 in the upper triangular half, modified by -1 at every $(i, j)$ with $|\{i, j\} \oplus S|=1$. Valiant's Pfaffian Sum Theorem in [5] states that $G^{S}=\operatorname{PfS}(B)=$ $\operatorname{Pf}\left(B+\Lambda^{(n)}(S)\right)$.

If $n$ is odd, then $G=H_{2}^{\otimes n} \Gamma$ is also expressed as a Pfaffian Sum. We define $B^{+}$to be the $(n+1) \times(n+1)$ matrix of which the first $n$ rows and columns equal $B$, and the $(n+1)$ st row and column are all 0 . Extend $\Lambda^{(n)}(S)$ to $\Lambda^{(n+1)}(S)$ with $\lambda_{n+1}=1$. Then $G^{S}=\operatorname{PfS}(B)=\operatorname{Pf}\left(B^{+}+\Lambda^{(n+1)}(S)\right)$.

Finally, suppose $\Gamma^{11 \ldots 1}=0$. If $\Gamma$ is identically 0 , then it can be represented by the Pfaffian of an all zero matrix. Suppose $\Gamma$ is not identically 0 , and $\Gamma^{T_{0}}=\lambda \neq 0$, for some $T_{0} \subset[n]$. We can define a modified matchgate $\tilde{\Gamma}$ extending the $i$-th external node $x_{i}$ by an edge $\left(x_{i}, x_{i}^{\prime}\right)$ of weight one, making $x_{i}^{\prime}$ the new external node, for every $i \notin T_{0}$. Also add one isolated edge with weight $1 / \lambda$. Then the signature of $\tilde{\Gamma}$ satisfies $\Gamma^{T}=\lambda \tilde{\Gamma}^{T \oplus T_{0}^{c}}$, and $\tilde{\Gamma}^{11 \ldots 1}=\Gamma^{T_{0}} / \lambda=1$. Now we can apply the construction above to matchgate $\tilde{\Gamma}$. More specifically, define $\tilde{B}$ from $\tilde{\Gamma}$, and $\Lambda^{(n)}(S)$ as before. Let $G=H_{2}^{\otimes n} \Gamma$, then for $n$ even,

$$
\begin{aligned}
G^{S} & =\sum_{T \subseteq[n]}(-1)^{|S \cap T|} \Gamma^{T} \\
& =\lambda \sum_{T \subseteq[n]}(-1)^{|S \cap T|} \tilde{\Gamma}^{T \oplus T_{0}^{c}} \\
& =\lambda \sum_{T^{\prime} \subseteq[n]}(-1)^{\left|S \cap T^{\prime}\right|}(-1)^{\left|S \cap T_{0}^{c}\right|} \tilde{\Gamma}^{T^{\prime}} \\
& =\lambda(-1)^{\left|S \cap T_{0}^{c}\right|} \operatorname{Pf}\left(\tilde{B}+\Lambda^{(n)}(S)\right) .
\end{aligned}
$$

For $n$ odd, the same result follows using $\tilde{B}^{+}$and $\Lambda^{(n+1)}(S)$ with $\lambda_{n+1}=1$.

We summarize the discussion as follows:

Theorem 0.1 A signature $G$ is realizable as a matchgate signature under the Hadamard transformation $\mathrm{H}_{2}$ iff it can be parameterized by an $n \times n$ skew-symmetric matrix as follows. If $n$ is even, then there exist $\lambda \in \mathbb{C}, T_{0} \subseteq[n]$, and $n \times n$ skewsymmetric matrix $B$, such that for all $S \subseteq[n]$,

$$
G^{S}=\lambda(-1)^{\left|S \cap T_{0}^{c}\right|} \operatorname{Pf}\left(B+\Lambda^{(n)}(S)\right)
$$

where $\Lambda^{(n)}(S)$ is defined in (2). If $n$ is odd, then it is suitably modified as described above

$$
G^{S}=\lambda(-1)^{\left|S \cap T_{0}^{c}\right|} \operatorname{Pf}\left(B^{+}+\Lambda^{(n+1)}(S)\right) .
$$

\section{References}

1. Cai, J-Y., Choudhary, V.: Some results on matchgates and holographic algorithms. In: Proceedings of ICALP 2006, Part I. Lecture Notes in Computer Science vol. 4051. pp 703-714. Int. J. Softw. Info. 1(1), 3-36 (2007) 
2. Cai, J-Y., Choudhary, V., Lu, P.: On the theory of matchgate computations. In Proceedings of CCC '07: Proceedings of the Twenty-Second Annual IEEE Conference on Computational Complexity, 2007, pp. 305-318. Theory Comput. Syst. 45(1):108-132 (2009)

3. Cai, J-Y.: Aaron Gorenstein. Matchgates Revisited. Theory of Computing (ToC) Volume 10, Article 868, pp. 401-430 (2014)

4. Cai, J.-Y., Lu, P.: Signature theory in holographic algorithms. Algorithmica 61(4), 779-816 (2011)

5. Valiant, L.G.: Quantum circuits that can be simulated classically in polynomial time. SIAM J. Comput. 31(4), 1229-1254 (2002) 